\title{
Proximate Composition, Polyphenol Content and Anti-inflammatory Properties of White and Pigmented Italian Rice Varieties
}

\author{
Katia Petroni $^{1, *}$, Michela Landoni ${ }^{1}$, Federica Tomay ${ }^{1}$, Valentina Calvenzani ${ }^{1}$, \\ Cinzia Simonelli ${ }^{2}$, Mauro Cormegna ${ }^{2}$ \\ ${ }^{1}$ Dipartimento di Bioscienze, Università degli Studi di Milano, Italy \\ ${ }^{2}$ Ente Nazionale Risi, Laboratorio Chimico Merceologico, Centro Ricerche sul Riso, Italy
}

Copyright $\mathrm{C} 2017$ by authors, all rights reserved. Authors agree that this article remains permanently open access under the terms of the Creative Commons Attribution License 4.0 International License

\begin{abstract}
Rice (Oryza sativa L.) is a major staple food for the majority of the world population. Grown and harvested as paddy, it is present on our tables as white rice (milled), brown rice (dehusked) or, after being subjected to thermal processes, as parboiled rice. Recently, pigmented varieties are also appreciated (red and black rice). In this study, we evaluated proximate composition, polyphenol content and anti-inflammatory properties of Italian pigmented rice varieties and bran as well as white rice in different processed forms (milled, parboiled and integral), in order to assess the nutritional and antioxidant properties of Italian rice varieties and their capacity to reduce inflammation. Our results showed that pigmented rice varieties displayed high antioxidant capacity along with the highest flavonoid and polyphenol content. Anthocyanins were mainly present in black rice, whereas alkylresorcinols were found only in red rice. Rice bran proved to be particularly rich in carotenoids. The evaluation of the anti-inflammatory properties using lipopolysaccharide (LPS)-induced mouse RAW 264.7 macrophages pre-treated with extracts obtained from integral, black, red rice and bran showed that the expression of inducible Nitric Oxide Synthase (iNOS) was specifically reduced at both transcript and protein level by extracts, indicating the capacity of rice extracts to modulate the inflammatory response.
\end{abstract}

Keywords Rice, Nutritional Properties, Antioxidant Capacity, Anti-inflammatory Properties

\section{Introduction}

Rice is the third most important cereal food in the world and the staple food in many Asian countries. The most common rice consumed by humans is white rice (about $85 \%$ ), but an increasing interest emerged around pigmented rice varieties, such as black, red and dark purple rice, due to the considerable amount of polyphenols present in the external layers of rice kernels. Black and purple rice mainly contain anthocyanins, a specific class of flavonoids conferring red to purple color [1], whereas pro-anthocyanidins are the main polyphenols in red rice [2]. Several beneficial properties, such as antioxidant, antitumoral, anti-atherogenic, anti-obesity, hypoglycemic and antiallergic effect have been reported for pigmented rice, especially black and dark purple rice [3]. Pigmented rice is popular and consumed mostly in Asian countries and the properties of many Asian rice varieties have been characterised so far [3]. On the other hand, the knowledge around the nutritional and health-promoting properties of Italian white and pigmented rice varieties is currently very limited $[4,5]$.

Rice is mainly consumed as milled rice, obtained by removing the hull and bran layer from the rice kernel. After removal of husk, rice is referred to as brown rice, which consists of bran (6-7\% by weight), endosperm (about $90 \%$ ) and embryo (2-3\%) [6]. White rice, also referred to as milled or polished rice, is obtained by removing $8-10 \%$ of external layers (mainly bran) from brown rice [7]. Although the polishing process provides benefits to the physical and sensorial properties of rice, it depletes rice of most nutrients and phytochemicals present in the kernels [8]. Rice bran is the main by-product derived from rice milling and is rich in proteins, fibers, vitamins, fats and polyphenolic compounds, which are mainly located in the external layers of the rice kernel [9]. Rice bran has recently received considerable attention due to its beneficial health effects against diabetes, cancer and heart disease [3]. Its use as largely available and inexpensive source of natural antioxidants and bioactive peptides with health-promoting properties has been recently evaluated $[10,11]$.

Parboiling of rice is an effective processing technique to 
increase storage stability of rice with minimal changes in nutritional properties. It occurs before dehusking and consists of soaking, pressure steaming and drying, after which rice undergoes conventional processing. During parboiling, the transfer of bran components to the inner layers of rice kernels, the inactivation of lipase and starch retrogradation have been widely documented [12]. Interestingly, parboiling of pigmented rice has been recently shown to allow the partial preservation of free phenolics, which would be otherwise lost in polishing process of rice [8].

Rice has been considered a source of carbohydrate with low to moderate dietary fiber content, based on the analysis of total dietary fiber and resistant starch. The amount of resistant starch varies in different rice cultivars, depending on the amylose content, and it can be increased by the parboiling process [13]. Due to its chemical nature and low digestibility, resistant starch is considered a type of dietary fiber. Resistant starch is the fraction of starch, resistant to enzyme hydrolysis, entering the large intestine along with dietary fibers. As part of dietary fiber, resistant starch is fermented by the gut microbiota to produce short chain fatty acids that signal satiety and impact insulin signalling [14]. Thus, resistant starch from rice has the potential to improve human health; nevertheless its content in commonly consumed rice cultivars or pigmented rice varieties needs further characterization.

Inflammation represents the main response against injury and it is normally characterised by an early acute phase, followed by the resolution of inflammation [15]. This process involves various cell types, including leukocytes that are recruited to the site of injury through chemotaxis. Failure in promoting resolution results in the establishment of a chronic state of inflammation, which represents a risk factor of various types of diseases, including cancer, diabetes, cardiovascular and autoimmune diseases [15]. Therefore, dietary strategies to reduce acute inflammation and prevent its degeneration towards the chronic state are attracting great interest.

The present study aimed at characterizing the proximate composition, polyphenol content and anti-inflammatory properties of Italian pigmented rice varieties and bran as well as white rice in different processed forms (milled, parboiled and integral), in order to assess the nutritional and antioxidant properties of Italian rice varieties and their capacity to reduce inflammation.

\section{Materials and Methods}

\subsection{Materials}

The current Italian legislation provides that all rice varieties suitable for trade are listed in an annual ministerial decree (currently valid: DM 30 September 2016, No 275, GU $24 / 11 / 2016$ ), divided into product groups. Based on this classification, the following Italian rice varieties from Ente Nazionale Risi germplasm were analyzed: a typical white variety suitable for cooking the risotto in three commercially available forms (milled rice, parboiled rice and integral rice), a black variety, a red variety and a mixture of rice bran kindly provided by Euricom Spa (Vercelli, Italy).

\subsection{Proximate Analysis}

Protein content was calculated from nitrogen content assessed by the Kjeldahl method using a 5.95 conversion factor. A test portion of milled rice $(1 \mathrm{~g})$ was digested by sulfuric acid $18 \mathrm{~mol} / \mathrm{L}(20 \mathrm{~mL})$ in the presence of a catalyst. The reaction products was made alkaline, and then distilled. The liberated ammonia was collected in a boric acid solution, which was titrated with a sulfuric acid solution, to determine the nitrogen content and calculate the crude protein content according to ISO 20483:2006 [16]. For crude fat, the Soxhlet extraction method with petroleum ether as solvent was used, according to AACC Method 30-25.01 [17]. Briefly, 5g of dried (in a vacuum oven: $100 \mathrm{~mm} \mathrm{Hg} ; 5 \mathrm{~h}$ ) grinded rice were extracted with $100 \mathrm{~mL}$ of petroleum ether $\left(30-60^{\circ} \mathrm{C}\right)$ for $2 \mathrm{~h}$. Moisture content was determined by using the standard methods of analysis ISO 712:2009 [16] and expressed as percentage. In particular, $5 \mathrm{~g}$ of grinded rice were dried in a Memmert UFE 400 oven for 2hs. Ash content was determined according to the AACC method 08-01 [17]. After determining the amount of dietary fiber and subtracting it from total carbohydrate, available carbohydrates were estimated by difference as follows: 100 - weight in grams [protein + fat + moisture + ash + dietary fiber] in $100 \mathrm{~g}$ of rice sample. Dietary fiber was determined according to method AOAC 985.29 [18].

\subsection{Determination of Total and Resistant Starch and Amylose Content}

The amount of total and resistant starch were determined following the AACC Method 32-40.01 using the K-RSTAR kit (Megazyme, Wicklow, Ireland), according to manufacturer's instructions. Rice flour $(100 \mathrm{mg})$ was incubated with $4 \mathrm{~mL}$ pancreatic $\alpha$-amylase $(10 \mathrm{mg} / \mathrm{mL})$ containing amyloglucosidase $(3 \mathrm{U} / \mathrm{mL})$ in a shaking water bath at $37^{\circ} \mathrm{C}$ for $16 \mathrm{~h}$. Then, ethanol $(4 \mathrm{~mL}, 99 \% \mathrm{v} / \mathrm{v})$ was added and samples were centrifuged at $2000 \mathrm{~g}$ for 10 mins. Pellets were resuspended with $2 \mathrm{~mL}$ ethanol $(50 \% \mathrm{v} / \mathrm{v})$ and, after adding $6 \mathrm{~mL}$ ethanol $(50 \% \mathrm{v} / \mathrm{v})$, samples were centrifuged at $2000 \mathrm{~g}$ for $10 \mathrm{mins}$. Pellets were then resuspended and centrifuged with the same procedure. To measure the resistant starch content, pellets resuspended by stirring with $2 \mathrm{~mL}$ potassium hydroxide $(2 \mathrm{M})$ and incubated in an ice water-bath for $20 \mathrm{~min}$. After adding $8 \mathrm{~mL}$ of sodium acetate buffer (1.2 M, pH 3.8) and $0.1 \mathrm{~mL}$ amyloglucosidase $(3300 \mathrm{U} / \mathrm{mL})$, samples were incubated in a water bath at $50^{\circ} \mathrm{C}$ for $30 \mathrm{~min}$ and then centrifuged at $1500 \mathrm{~g}$ for $10 \mathrm{~min}$. Aliquots of supernatants $(0.1 \mathrm{~mL})$ were incubated into glass 
test tubes with glucose oxidase/peroxidase (GOPOD) reagent in a water bath at $50^{\circ} \mathrm{C}$ for $20 \mathrm{~min}$. Absorbance was immediately measured at $510 \mathrm{~nm}$ compared to the following reagent blank $(0.1 \mathrm{~mL}$ of $100 \mathrm{mM}$ sodium acetate buffer $\mathrm{pH}$ 4.5 and $3 \mathrm{~mL}$ of GOPOD reagent). To measure the digestible starch, the supernatants obtained by the three initial centrifugations were combined, the volume adjusted to 100 $\mathrm{mL}$ with $100 \mathrm{mM}$ sodium acetate buffer $\mathrm{pH} 4.5$ and a $0.1 \mathrm{~mL}$ aliquot was incubated with $10 \mu \mathrm{L}$ of amyloglucosidase solution $(300 \mathrm{U} / \mathrm{mL})$ for $20 \mathrm{~min}$ at $50^{\circ} \mathrm{C}$. After adding $3 \mathrm{~mL}$ of GOPOD reagent, samples were incubated at $50^{\circ} \mathrm{C}$ for 20 min. The absorbance was measured at $510 \mathrm{~nm}$ against a reagent blank.

The resistant and non-resistant starch content was calculated using the Megazyme Mega-Calc ${ }^{\mathrm{TM}}$ program (Megazyme, Wicklow, Ireland). The total starch content is the sum of resistant and non-resistant starch content. The starch content was expressed as $\mathrm{g} / 100 \mathrm{~g}$ of rice flour.

For the determination of amylose content, rice was ground to a very fine flour (Retsch ZM200 grinding system and sieving with a $150 \mu \mathrm{m}$ Endecotts sieve) to break up the endosperm structure in order to aid complete dispersion and gelatinization; the flour was then defatted. A test portion was dispersed in a sodium hydroxide solution, to an aliquot portion of which an iodine solution was added. The absorbance, at $720 \mathrm{~nm}$, of the color complex formed was then determined using a UV-VIS spectrophotometer (Lambda 25, Perkin Elmer). The amylose mass fraction of the sample was then read with a calibration graph, which was prepared using mixtures of potato amylose and amylopectin to make allowance for the effect of amylopectin on the colour of the amylose-iodine complex of the test solution, according to the ISO 6647-1:2007 method [16].

\subsection{Determination of Antioxidant Capacity and Total Polyphenol, Flavonoid and Anthocyanin Content}

Methanolic extracts were obtained from $0.5 \mathrm{~g}$ of rice flours with $20 \mathrm{~mL}$ of methanol- $1 \% \mathrm{HCl}$ and used according to the procedures described below.

The antioxidant capacity was measured by determining the ability of methanolic extracts to scavenge 2,2 azinobis (3-ethylbenzothiazoline-6-sulfonic acid) (ABTS). The ABTS radical scavenging activity was measured by using the TEAC (Trolox Equivalent Antioxidant Capacity) method as previously described [19] and expressed as $\mu \mathrm{mol}$ of Trolox equivalents (TE) per $100 \mathrm{~g}$ of rice flour.

The total phenolic content was determined using the Folin-Ciocalteau method. Methanolic extracts were mixed with Folin-Ciocalteau's phenol reagent $0.2 \mathrm{~N}$ (in a ratio of 1:5). Sodium carbonate (7.5\%) was added into the mixtures (in a ratio of 1.5:1) and incubated for $2 \mathrm{~h}$. The reaction mixture was then read at $760 \mathrm{~nm}$ against a standard curve of gallic acid and total polyphenol content was reported as $\mathrm{mg}$ of gallic acid (GA) equivalents per $100 \mathrm{~g}$ of rice flour.

To measure flavonoid content, methanolic extracts were incubated with $2 \% \mathrm{AlCl}_{3}$ for $25 \mathrm{~min}$ and then read at $510 \mathrm{~nm}$ against a standard curve of quercetin. The flavonoid content was expressed as mg quercetin $(\mathrm{Qc})$ equivalent $/ 100 \mathrm{~g}$ of rice flour. With this method it is possible to determine the total content of flavonoids, including chalcones, flavones, flavonols, and anthocyanins.

The total anthocyanin content was measured with the pH-differential method. Briefly, $1 \mathrm{~mL}$ of the methanolic extracts was treated separately with $4 \mathrm{~mL}$ of $\mathrm{pH} 1.0$ buffer $(25 \mathrm{mM} \mathrm{KCl} \mathrm{pH} \mathrm{1)} \mathrm{or} 4 \mathrm{~mL}$ of $\mathrm{pH} 4.5$ buffer $(400 \mathrm{mM}$ sodium acetate $\mathrm{pH} 4.5$ ). The samples were thoroughly mixed and the absorbance was measured at $520 \mathrm{~nm}$ and $700 \mathrm{~nm}$. The total anthocyanin content was calculated according to the equation:

Amount $\left[\mathrm{mg} \mathrm{g}^{-1}\right]=\Delta \mathrm{A} \times 449.2 / 26900 \times$ dilution factor $\mathrm{x}$ final volume $(\mathrm{mL}) /$ sample weight $(\mathrm{g})$ where $\Delta \mathrm{A}:\left(\mathrm{A}_{510}-\mathrm{A}_{700}\right)$ $\mathrm{pH} 1-\left(\mathrm{A}_{510}-\mathrm{A}_{700}\right) \mathrm{pH} 4.5$. The value 449.2 represents the molecular mass of cyanidin 3-glucoside, whereas 26900 is its molar absorbance. Each sample was analyzed in triplicate and the anthocyanin content expressed as $\mathrm{mg}$ cyanidin 3 -glucoside $(\mathrm{C} 3 \mathrm{G})$ equivalents per $100 \mathrm{~g}$ of rice flour.

\subsection{Determination of Alkylresorcinols}

To determine the alkylrercinol content, $0.2 \mathrm{~g}$ of rice flour was extracted with $8 \mathrm{~mL}$ of acetone, after $48 \mathrm{~h}$ of shaking the samples were centrifuged and the supernatant dried under vacuum and redissolved in $200 \mu \mathrm{L}$ of methanol. For the colorimetric reaction, $10 \mu \mathrm{L}$ of each sample were treated with $2 \mathrm{~mL}$ of Fast Blue Zn Salt $0.05 \%$ freshly diluted with 5 volumes of methanol. After incubation of $1 \mathrm{~h}$ in the dark, the samples were read at $520 \mathrm{~nm}$. The alkylresorcinol content was calculated using a calibration curve made with 5-pentadecylresorcynol (C21) as standard and expressed as $\mathrm{mg} / 100 \mathrm{~g}$ of rice flour.

\subsection{Determination of Carotenoid Content}

Carotenoids were extracted as previously reported [20]. Briefly, $6 \mathrm{~mL}$ of methanol, acetone and hexane $(1: 1: 1, \mathrm{v} / \mathrm{v})$ were added to a glass tube containing a sample of $0.5 \mathrm{~g}$ of rice flour and shaken horizontally for $30 \mathrm{~min}$. After centrifugation, $1 \mathrm{~mL}$ of the non-polar phase containing carotenoids was filtrated through a $0.22 \mu \mathrm{m}$ filter and analyzed spectrophotometrically at $448 \mathrm{~nm}$. The carotenoid content was calculated from the calibration curve of lutein as standard and expressed as $\mu \mathrm{g}$ lutein equivalents per $100 \mathrm{~g}$ of rice flour.

\subsection{Cell Culture}

Mouse RAW 264.7 macrophages were purchased from Sigma Aldrich (91062702-1VL) and cultured in DMEM (Sigma), supplemented with 10\% fetal bovine serum (Sigma), $100 \mu \mathrm{g} / \mathrm{mL}$ penicillin/streptomycin (Sigma) and $2 \mathrm{mM}$ L-glutamine (Sigma). Cells were maintained at $37^{\circ} \mathrm{C}$ in $5 \%$ 
$\mathrm{CO}_{2}$ atmosphere. The cells were in the exponential phase of growth before exposure to extracts in all experiments.

Rice extracts were prepared from $12 \mathrm{~g}$ of each rice flour (i.e. integral, red, black rice and bran) with $20 \mathrm{~mL} / \mathrm{g}$ of $\mathrm{n}$-hexane and the mixture was shaken at $250 \mathrm{rpm}$ for $30 \mathrm{~min}$ at room temperature. After centrifuging at $10000 \mathrm{~g}$ for 10 min, the residue was dried at room temperature in a fume hood and then extracted twice with methanol- $1 \% \mathrm{HCl}$. After shaking at $250 \mathrm{rpm}$ for $45 \mathrm{~min}$ per extraction, the mixture was centrifuged at $10000 \mathrm{~g}$ for $15 \mathrm{~min}$. The supernatants of each methanol extraction were combined, concentrated at equal volumes using a vacuum refrigerated centrifuge and stored at $-20{ }^{\circ} \mathrm{C}$ for further analyses.

Prior to the experiments, RAW 264.7 cells were plated at a density of 35000 cells/well in 96-well plates and cultured for $48 \mathrm{~h}$. The cells were treated for $48 \mathrm{~h}$ with different concentrations of methanol extracts $(0,5,25,125,250 \mu \mathrm{M})$. Cell viability was determined using 3-(4,5-dimethylthiazol-2-il)-2,5-diphenyltetrazolium bromide (MTT) test. Briefly, $10 \%$ of MTT solution per volume was added to each well and incubated for additional $4 \mathrm{~h}$. Formazan crystals were dissolved in acidified isopropanol (isopropanol, $0.1 \mathrm{~N} \mathrm{HCl}, 0.1 \%$ Tween). The optical density was measured at $570 \mathrm{~nm}$ wavelength, by using a microplate reader (Tecan Infinite F200PRO).

RAW 264.7 macrophages were plated at a density of $1 \times 10^{6}$ cells/well in 6-well plates and grown for $24 \mathrm{~h}$. The cells were pre-treated with the chosen dosage of extracts $(125 \mu \mathrm{M})$ for $16 \mathrm{~h}$ and then $100 \mathrm{ng} / \mathrm{mL}$ LPS (Sigma L4391) was added for additional $6 \mathrm{~h}$ for RNA extraction or $24 \mathrm{~h}$ for supernatants and protein analysis.

\subsection{RNA Extraction and qRT-PCR Analysis}

Table 1. Primer sets used for qRT-PCR analysis

\begin{tabular}{|c|c|c|}
\hline \multirow{2}{*}{ Gene } & Primer name & Sequences $\left(5^{\prime} \rightarrow 3^{\prime}\right)$ \\
\hline \multirow{2}{*}{$I L-1 \beta$} & TNFa-F & GGAGTTTGAGTCTGCAGAGTTCC \\
\cline { 2 - 3 } & TNFa-R & GGGGAAGGCATTAGAAACAGTCC \\
\cline { 2 - 3 } & mIL1bfor & GGTGTGTGACGTTCCCATTA \\
\hline \multirow{2}{*}{$I L-6$} & mIL6for & CAGAGTCCTTCAGAGAGATACA \\
\cline { 2 - 3 } & mIL6rev & CCTTCTGTGACTCCAGCTTATC \\
\hline \multirow{2}{*}{$i N O S$} & miNOSfor & TGGTGTCTGCAGCACTTGGA \\
\cline { 2 - 3 } & miNOSrev & TTGAGCCCTTTGTGCTGGGA \\
\hline \multirow{2}{*}{$C O X-2$} & mCOX2for & CGAGTCGTTCTGCCAATAGAA \\
\cline { 2 - 3 } & mCOX2rev & CCTGGTCGGTTTGATGTTACT \\
\hline \multirow{2}{*}{$G A P D H$} & GAPDH-Fw & ATGTGTCCGTCGTGGATCTG \\
\hline & GAPDH-Rv & GGTGGAAGAGTGGGAGTTGC \\
\hline
\end{tabular}

Total RNA was extracted using Direct-zol ${ }^{\mathrm{TM}}$ RNA Miniprep kit (Zymo Research). Synthesis of cDNA was obtained using the RT SuperscriptTM II (Invitrogen,
Carlsbad, CA). Transcript analysis was performed using SOS Fast EVA-Green Supermix (BioRad Laboratories, Hercules, CA) on a Cfx96 9 TMBioRad Real Time system. Values were normalized on transcript level of the $G A P D H$ housekeeping gene and expressed as Fold Change (FC) relative to untreated control sample. Primer sequences are indicated in Table 1.

\subsection{Enzyme-Linked Immunosorbent Assay (ELISA)}

Supernatants were analyzed for mIL6, mIL1 $\beta, \operatorname{mTNF} \alpha$ and mIL10 using Ready SET-Go! ELISA kit (Affymetrix) according to the manufacturer's instructions. The optical density (O.D.) of each well was analyzed at $450 \mathrm{~nm}$ by a Tecan Microplate Reader (Tecan).

\subsection{Western Blot Analysis}

Total proteins were extracted using RIPA buffer $(10 \mathrm{mM}$ Tris-HCl pH 8.0, 1 mM EDTA, 0.5 mM EGTA, $1 \%$ Triton $\mathrm{X}-100,0.1 \%$ sodium deoxycholate, $0.1 \%$ SDS, $140 \mathrm{mM}$ $\mathrm{NaCl}, 1 \mathrm{mM}$ PMSF), lysates were centrifuged and the supernatants were collected. Equal amounts of proteins (30 $\mu \mathrm{g})$ underwent SDS-PAGE and were transferred to nitrocellulose membranes. Membranes were incubated with primary antibodies against Anti-iNOS (Cayman chemical, ab160862) or $\alpha$-Tubulin (Sigma, T6074) and probed with horseradish peroxidase-conjugated secondary antibodies. Bands were detected using Pierce ${ }^{\mathrm{TM}}$ ECL Western Blotting Substrate (ThermoFisher Scientific). All the antibodies were applied at 1:2000 dilutions.

\subsection{Statistical Analysis}

Analytical determinations for the samples were performed in triplicate on at least three biological samples. All values were expressed as means \pm SEM, except where specified. Data analysis was carried out by one way ANOVA and Tukey's means comparison test with GraphPad Prism version 6.0 (GraphPad Software). Differences were considered significant when $\mathrm{p}<0.05$.

\section{Results and Discussion}

\subsection{Proximate Composition}

The proximate compositions of white rice in three processed forms (milled, integral, and parboiled), red and black rice as well as bran are presented in Table 2. The moisture content of all three Italian varieties, including different processed forms of white rice, and bran were lower than $14 \%$, which is considered safe for storing grains to avoid damage and deterioration of seed quality [9]. 
Table 2. Proximate composition of milled, parboiled and integral rice, red and black rice, bran

\begin{tabular}{|c|c|c|c|c|c|c|}
\hline Component (\%) & Milled & Parboiled & Integral & Red & Black & Bran \\
\hline Moisture & $10.87 \pm 0.15$ & $11.94 \pm 0.32$ & $10.72 \pm 0.24$ & $10.78 \pm 0.19$ & $10.59 \pm 0.28$ & $9.11 \pm 0.32$ \\
\hline Carbohydrates & $81.24 \pm 7.90$ & $79.28 \pm 7.48$ & $75.04 \pm 6.80$ & $71.20 \pm 6.85^{\mathrm{c}}$ & $73.39 \pm 7.12^{\mathrm{c}, \mathrm{d}}$ & $27.45 \pm 2.47^{\mathrm{a}}$ \\
\hline Proteins & $5.78 \pm 0,52$ & $5.57 \pm 0,53$ & $6.24 \pm 0,58$ & $7.88 \pm 0,69$ & $7.37 \pm 0,80$ & $12.4 \pm 0.95^{\mathrm{a}}$ \\
\hline Lipids & $0.93 \pm 0.05$ & $1.11 \pm 0.04$ & $2.91 \pm 0.50$ & $4.32 \pm 0.54$ & $3.16 \pm 0.14$ & $19.08 \pm 0.90^{\mathrm{a}}$ \\
\hline Ash & $0.29 \pm 0.06$ & $0.67 \pm 0.06$ & $1.25 \pm 0.09$ & $1.42 \pm 0.09$ & $1.58 \pm 0.08$ & $8.86 \pm 0.89^{\mathrm{b}}$ \\
\hline Dietary fiber & $0.89 \pm 0.08$ & $1.43 \pm 0.19$ & $3.84 \pm 0.34$ & $4.40 \pm 0.39$ & $3.91 \pm 0.35$ & $23.10 \pm 2.07^{\mathrm{a}}$ \\
\hline
\end{tabular}

Means of three determinations with standard deviations. Bran values were significantly different from all other rice samples at $\mathrm{p}<0,001$ (a) or $\mathrm{p}<0,05$ (b). Red and black rice values were significantly different from milled rice (c) or from parboiled rice (d) at $p<0,05$.

No significant differences were observed in carbohydrate content of milled, parboiled and integral rice ranging from $75.04 \%$ to $81.24 \%$, whereas in red and black rice carbohydrates were significantly lower than milled and parboiled rice (Table 2). Bran had a significantly lower content of carbohydrate, whereas lipid, ash and dietary fiber content was significantly higher compared to all other rice (Table 2). This was expected, since bran is mainly constituted of external layers of rice kernels, where proteins, lipids and dietary fiber are mostly concentrated [9].

Despite differences among the other rice samples were not statistically significant, content of lipids, ash, proteins and dietary fibers were lower in milled and parboiled rice, whereas integral, red and black rice showed higher content of such components, as a consequence of a lower degree of milling (Table 2). In particular, lipids can contribute to the quality of rice, influencing the nutritional and sensory aspect, although not abundant such as carbohydrates and proteins [21]. Lipid content in milled and parboiled rice was $0.93-1.11 \%$, whereas in integral, red and black rice it ranged from 2.91 to $4.32 \%$ (Table 2). Accordingly, the total concentration of lipids in the caryopsis of unmilled rice has been reported between 2 and $4 \%$, while concentration in the grain milled rice amounts to 0.3 to $0.6 \%$ [22]. Similarly, protein content in milled and parboiled rice was lower (5.57-5.78\%) compared to integral, red and black rice (6.24-7.88\%) (Table 2). Proteins play an important role in cooked rice texture, because they form a complex with starch that impairs starch granule swelling [21]. High protein rice is less sticky and has a harder texture, but rice obtained from a higher degree of milling has a comparatively better sensory quality [23].

The total dietary fiber content in milled and parboiled rice was found to range between $0.9-1.4 \%$, whereas it was higher in integral, red and black rice (3.8-4.4\%), in which more external layers of rice kernels are present (Table 2). Dietary fiber has been correlated to a reduced risk of type-2 diabetes and cardiovascular diseases. It can reduce appetite and contribute to weight loss, since it is not digested in the small intestine, but is fermented by microbiota in the colon, where it produces short-chain fatty acids, such as acetate, butyrate and propionate, which are important for satiety signalling [14].

\subsection{Resistant Starch, Digestible Starch and Amylose Content}

The total starch content was $62-69 \%$ in milled, parboiled and integral rice, whereas red and black rice contained a significantly lower content of starch (about 60\%) compared to milled rice, probably due to a lower degree of milling and the consequent presence of external seed integuments (Figure 1A). Consistently, rice bran has a very low total starch content compared to milled rice, being mainly constituted of external layers of rice kernels (Figure 1A).

Most of starch present in rice consists of digestible starch (Figure 1B), but in parboiled rice resistant starch is significantly higher compared to milled rice and, despite not significant, a similar trend was observed for integral rice (Figure 1C). Foods with higher levels of resistant starch have been reported to reduce glucose and insulin responses and confer enhanced satiety as well as to reduce potential risk factors for type 2 diabetes and the metabolic syndrome [24].

Amylose content is considered the single most important determinant for predicting the eating quality of rice [23]. Amylose content is directly related to water absorption, volume expansion, fluffiness and separability of cooked rice. It is inversely related to cohesiveness, tenderness, glossiness and cooked grain stickiness, whereas it is positively correlated with cooked grain [23]. Based on amylose content, rice can be classified in 5 groups. Most Italian rice varieties are classified within Group III with a total amylose content ranging between 12 and $15 \mathrm{~g} / 100 \mathrm{~g}$, except some of them which are classified with Group IV with an amylose content of $25-27 \mathrm{~g} / 100 \mathrm{~g}$ [22]. Total amylose content of white rice in the three processed forms (milled, parboiled, integral), of red and black rice as well as bran is presented in Figure 1D. The total amylose content in milled rice was found to be approximately $17.4 \%$, thus classified under rice quality Group III [22]. Despite red and black rice contained a significantly lower amylose content compared to milled rice (approximately 9.9 and $10.5 \mathrm{~g} / 100 \mathrm{~g}$, respectively), they can also be classified within Group III (Figure 1D). Again, rice bran has only traces of amylose content compared to milled rice, being mainly constituted of external layers of rice kernels (Figure 1D). 

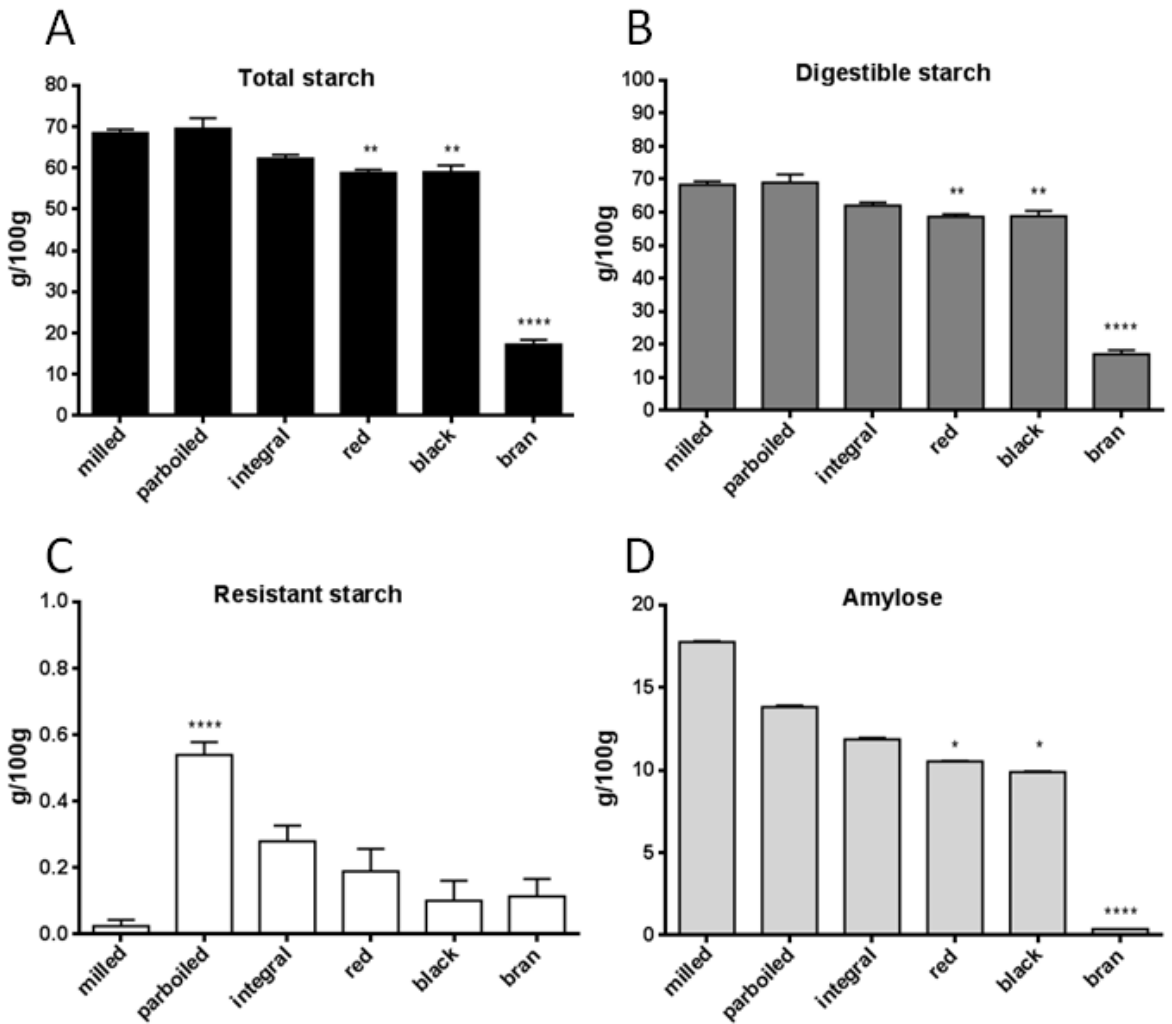

Figure 1. Determination of starch composition. Total, digestible and resistant starch (A-C) and amylose content (D) were determined in white rice in three different processed forms (milled, parboiled, integral) and in red, black rice and bran and expressed as $\mathrm{g} / 100 \mathrm{~g}{ }^{* * * *} \mathrm{p}<0.0001 ;{ }^{* * *} \mathrm{p}<0.001 ;{ }^{* *} \mathrm{p}<0.01$ indicate significant differences (one-way ANOVA) versus milled rice.

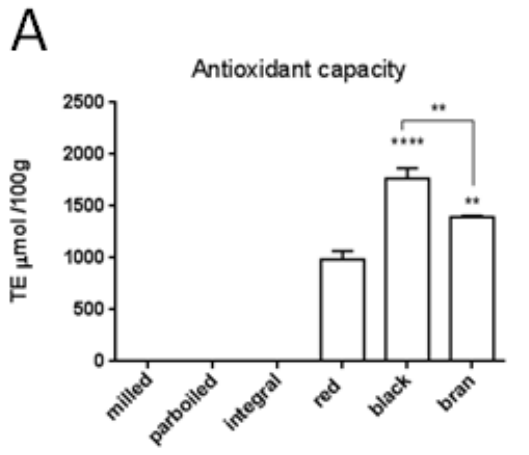

D

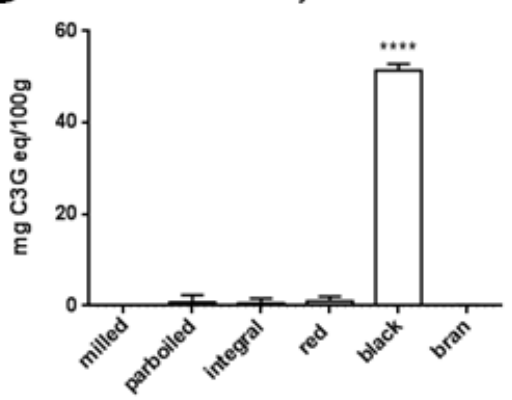

B

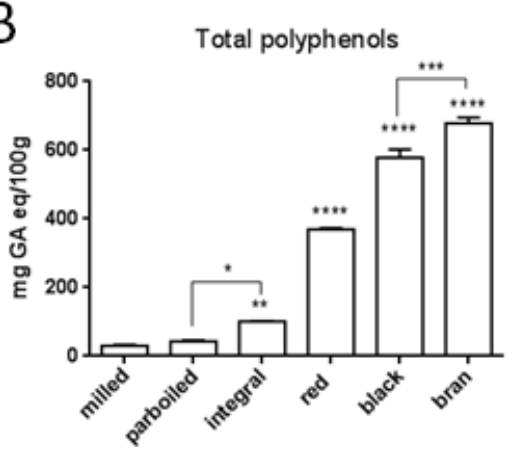

E

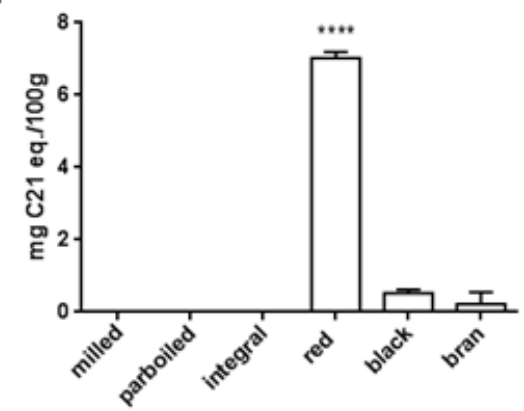

C

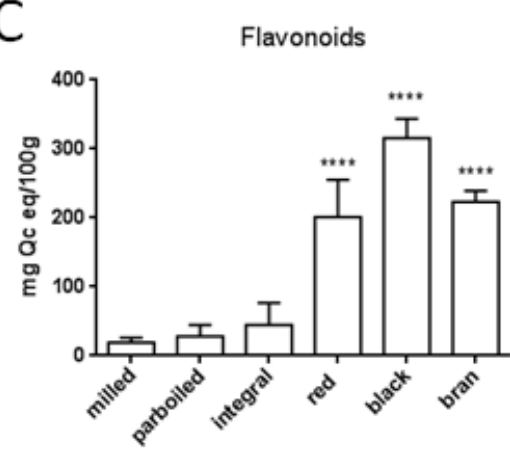

$\mathrm{F}$

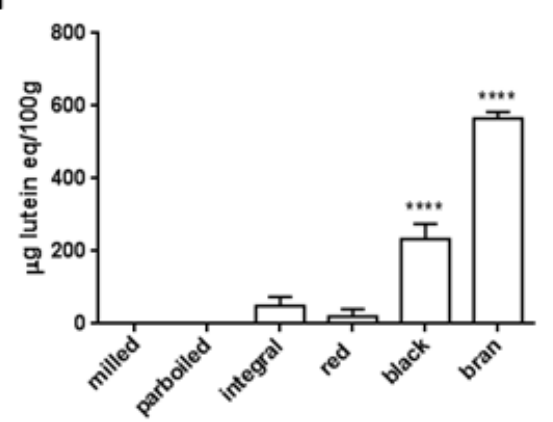

Figure 2. Determination of (A) antioxidant capacity expressed as $\mu$ mol of trolox equivalents (TE) per $100 \mathrm{~g}$, (B) total polyphenols expressed as mg of gallic acid (GA) equivalents per $100 \mathrm{~g}$, (C) flavonoids expressed as mg quercetin (Qc) equivalents per $100 \mathrm{~g}$, (D) anthocyanins expressed as mg cyanidin 3-glucoside (C3G) equivalents per $100 \mathrm{~g}$, (E) alkylresorcinols expressed as 5-pentadecyl resorcynol (C21) equivalents mg per $100 \mathrm{~g}$ and (F) carotenoids expressed as g lutein equivalents per 100 g. ${ }^{* * * *} \mathrm{p}<0.0001 ;{ }^{* * *} \mathrm{p}<0.001 ;{ }^{* *} \mathrm{p}<0.01 ;{ }^{*} \mathrm{p}<0.05$ indicate significant differences (one-way ANOVA) versus milled rice. 


\subsection{Total Polyphenol Content and Antioxidant Activity}

Milled, parboiled and integral rice did not show antioxidant activity, consistent with the almost complete removal of external integuments, whereas the two pigmented rice cultivars, especially black rice, and bran showed a significantly high antioxidant capacity (Figure 2A). Pigmented rice cultivars and bran showed higher total polyphenol content (372.7-678.0 mg gallic acid (GA) equivalents $/ 100 \mathrm{~g}$ ) than the remaining non-pigmented rice (30.5-101.3 mg GA equivalents /100g). Nonetheless, integral rice showed significantly higher polyphenol content compared to milled rice (Figure 2B). Higher levels of antioxidant activity of red, black rice and bran (984.20-1768 TE $\mu \mathrm{mol} / 100 \mathrm{~g})$ were strongly correlated with total polyphenol content (Figure 2A-B).

\subsection{Flavonoids, Anthocyanins, Alkylresorcinols and Carotenoids}

The analysis of the different classes of polyphenols associated with rice cultivars and bran showed that in integral rice polyphenols were mainly represented by flavonoids (Figure 2C), a small fraction of which consisted of anthocyanins (Figure 2D). Similarly, bran mainly contained flavonoids (Figure 2C) and a small quantity of anthocyanins and alkylresorcinols (Figure 2D,E). Concerning black rice, polyphenols were mainly represented by anthocyanins and to a smaller extent alkylresorcinols (Figure 2D,E), whereas in red rice a high level of alkylresorcinols was detected (Figure 2E). Significant differences in the amount of anthocyanins in black and red rice cultivars of different origins have been previously reported [1, 4, 25]. The data obtained from our analysis is consistent with these studies, since the anthocyanin content of black rice was $51.4 \mathrm{mg} / 100 \mathrm{~g}$, whereas that of red rice was only $0.98 \mathrm{mg} / 100 \mathrm{~g}$. It is known that pigmentation in red rice is mainly due to pro-anthocyanidins [2], but the presence of alkylresorcinols in pigmented rice cultivars has not been previously ascertained. High levels of alkylresorcinols were reported in wheat, rye and triticale and low amounts in barley, millet and maize. Although alkylresorcinols were found in rice seedlings, they were absent in kernels of rice [26]. Our data showed that alkylresorcinols were mainly present in red rice, but to a very low amount also in black rice and bran (Figure 2E). Alkylresorcinols have anticancer, antimicrobial and antioxidant activity in vitro and are mainly found in bran layers of cereal grains, which mean that they are lost in refined cereals [27]. Therefore, red rice may represent an additional source of these beneficial compounds, with an amount of alkylresorcinols $(7 \mathrm{mg} / 100 \mathrm{~g})$ similar to barley
(4.2-5.1 mg/100g) [26]. Interestingly, alkylresorcinols have been proposed as biomarkers of whole cereal grain intake when measured in plasma, in order to understand the preventive effect of whole grain consumption against chronic diseases [27].

The carotenoid content varied substantially among the different rice samples. Carotenoids were not detected in milled and parboiled rice, whereas the levels of carotenoids were 48.98, 20.59 and $232.8 \mu \mathrm{g} / 100 \mathrm{~g}$ in integral, red and black rice, respectively (Figure $2 \mathrm{~F}$ ). The total amounts in red and black rice were significantly lower from those reported in Korean or Camargue pigmented rice [25, 28], but the relative amount between red and black rice was similar, since the carotenoid content was 9-11 fold higher in black rice compared to red rice cultivars of similar origin. The carotenoid content found in bran was 11.6-fold higher than in integral rice and 2.4-fold higher than in black rice (Figure $2 \mathrm{~F}$ ), consistent with previous studies indicating that carotenoids are mainly located in rice bran [29].

\subsection{Anti-inflammatory Effect of Rice Extracts on LPS-induced RAW 264.7 Macrophage Cells}

In order to evaluate the anti-inflammatory effect of integral, red, black rice and bran, methanol extracts were prepared and a non-cytotoxic dose $(125 \mu \mathrm{M})$ determined by MTT assay, which showed no growth effects in RAW macrophage cells (data not shown), was used in later experiments.

Pathogen-induced acute inflammation induces activation of macrophages, resulting in production of pro-inflammatory cytokines, such as TNF- $\alpha$, IL- $1 \beta$ and IL-6 acting as inducers of acute phase reactions, but also an increase of nitric oxide (NO) production, regulated by inducible nitric oxide synthase (iNOS), and of COX-2 expression and its enzymatic product prostaglandin E2 (PGE2) [15]. Therefore, the expression of pro-inflammatory genes (i.e. TNF- $\alpha$, $I L-1 \beta, I L-6, i N O S$ and $C O X-2)$ was analyzed by qRT-PCR in LPS treated RAW 264.7 murine cell line. Cells were pre-incubated for $16 \mathrm{~h}$ with methanolic extracts from integral, red, black rice and bran and then treated with LPS for additional $6 \mathrm{~h}$. Transcript levels of all pro-inflammatory genes were strongly increased upon stimulation with LPS (Figure 3, CNT+LPS). Pre-treatment with rice extracts did not affect $T N F-\alpha, I L-6$ and $C O X-2$ genes (Figure $3 \mathrm{~A}, \mathrm{C}, \mathrm{E}$ ), whereas $I L-1 \beta$ transcript level increased with respect to CNT+LPS (Figure 3B). On the contrary, pre-treatment with rice extracts reduced the expression of $i N O S$ compared to CNT+LPS (Figure 3D). 

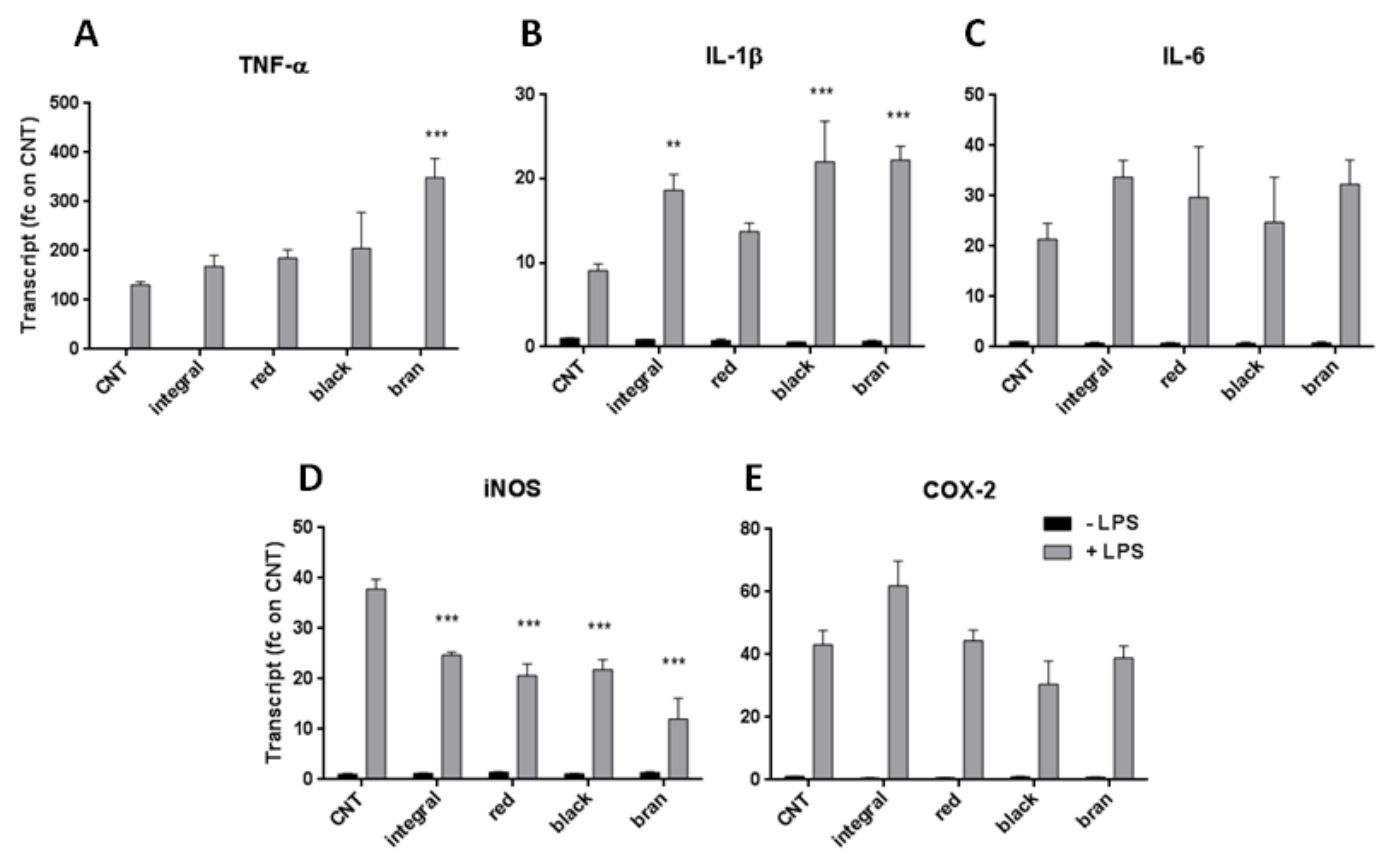

Figure 3. Expression levels of $T N F-\alpha, I L-1 \beta, I L-6, i N O S$ and $C O X-2$ transcripts in mouse RAW 264.7 macrophages pre-treated for $16 \mathrm{~h}$ with $125 \mu \mathrm{M}$ of extracts obtained from integral, red, black rice and bran followed by exposure to $100 \mathrm{ng} / \mathrm{mL}$ LPS for $6 \mathrm{~h}$. Each transcript was expressed as fold change compared to CNT-LPS. ${ }^{* *} \mathrm{p}<0.001 ; * \mathrm{*}<0.01$ indicate significant differences (one-way ANOVA) versus $\mathrm{CNT}+\mathrm{LPS}$.

A

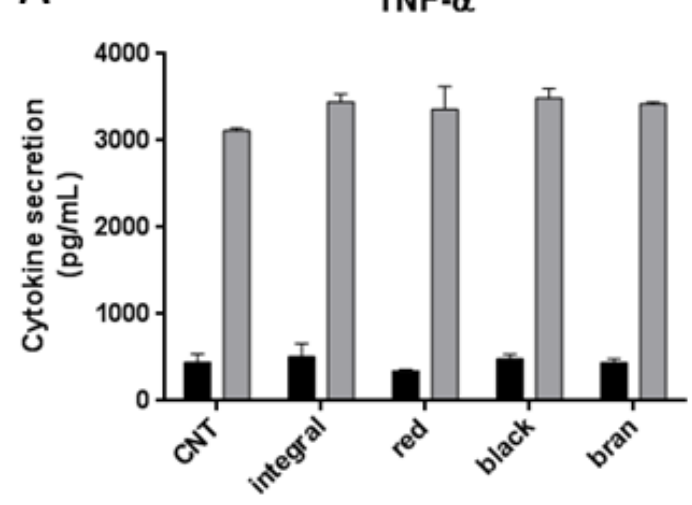

B

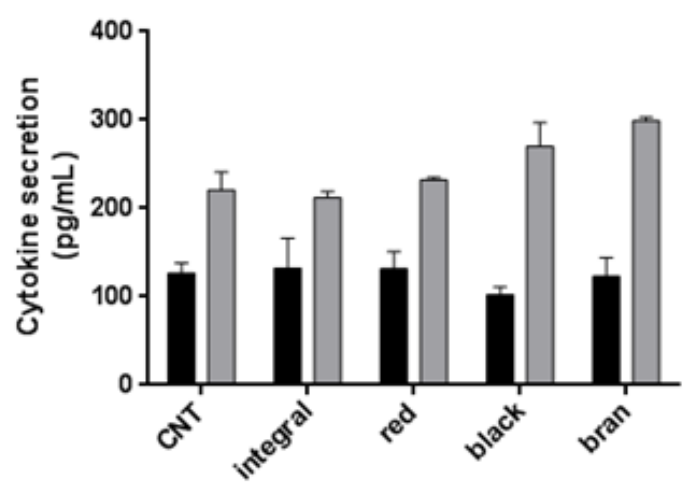

C

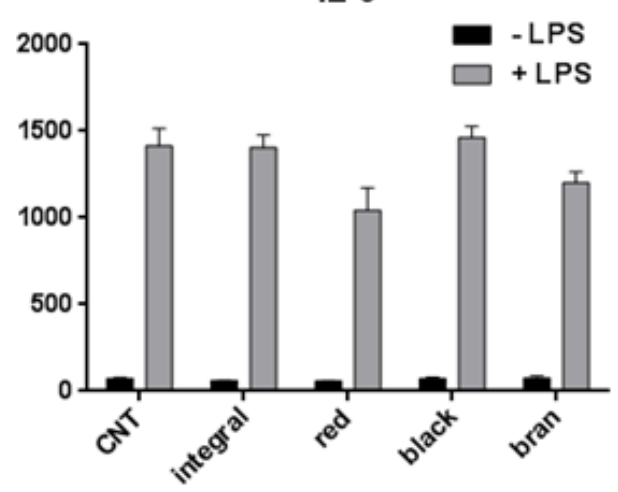

D

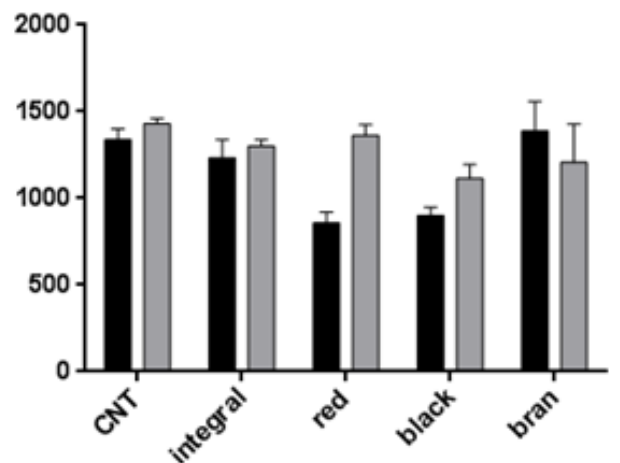

Figure 4. Secretion of pro-inflammatory and anti-inflammatory cytokines. Mouse RAW 264.7 macrophages pre-treated for $16 \mathrm{~h}$ with $125 \mu \mathrm{M}$ of extracts obtained from integral, red, black rice and bran followed by exposure to $100 \mathrm{ng} / \mathrm{mL}$ LPS for additional $24 \mathrm{~h}$. No significant differences were observed (one-way ANOVA) versus CNT +LPS. 


\subsection{Effect of Rice Extracts on Pro-inflammatory Cytokine Secretion and iNOs Protein Levels in LPS-induced RAW 264.7 Macrophage Cells}

We then analyzed the secretion of pro-inflammatory cytokines by ELISA to confirm the effects observed at the transcriptional level. The TNF- $\alpha$, IL- $1 \beta$ and IL- 6 secretions were all induced by LPS (Figure 4A-C) and remained unaffected by the pre-treatment with rice extracts, confirming that rice extracts do not prevent the release of pro-inflammatory mediators. In order to evaluate their potential effect on anti-inflammatory cytokines, the secretion of IL-10 by macrophages was evaluated. As shown in Figure $4 \mathrm{D}$, IL-10 secretion was not induced by LPS and pre-treatment of cells with rice extracts did not increase its expression compared to CNT+LPS, indicating that rice extracts have no effect on IL-10.

We then verified whether the reduced transcript level of the $i N O S$ gene correlated with a decrease of iNOS protein (Figure 5). The expression of iNOS protein was up-regulated in LPS-induced RAW macrophage cells. However, iNOS protein level was significantly decreased when the cells were pre-treated with rice extracts (Figure 5). Since no effect was observed on cytokines production, these results suggest that rice extracts exert their anti-inflammatory action by inhibiting iNOS, an inflammatory mediator regulating NO production upon infection. Similar findings of a flavonoid rich-extract reducing the expression of iNOS protein and corresponding transcript have been reported with extracts from black rice bran fraction or Ginko biloba in vitro [30,31]. A modest decrease of iNOS protein expression has been also observed in vivo in aortic tissue of ApoE mice fed with a black rice bran-supplemented diet [32]. Our in vitro study shows that a similar effect can be also obtained with extracts from other rice varieties in commercially available forms, such as integral rice, black and red rice, and a common by-product such as bran obtained from white rice varieties, suggesting it's possible as functional food.

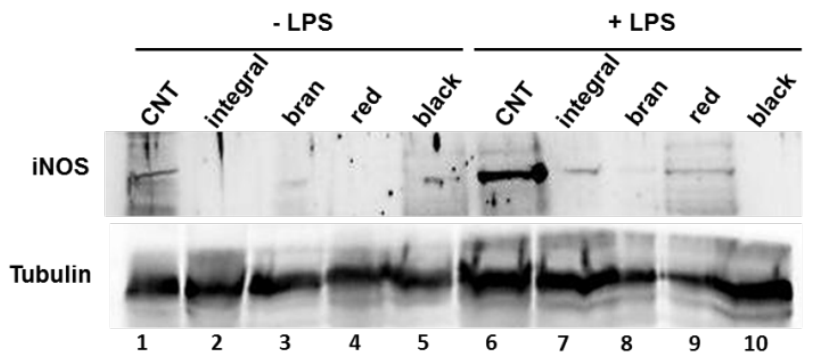

Figure 5. Expression level of iNOS protein. Mouse RAW 264.7 macrophages pre-treated for $16 \mathrm{~h}$ with $125 \mu \mathrm{M}$ of extracts obtained from integral, red, black rice and bran followed by exposure to $100 \mathrm{ng} / \mathrm{mL}$ LPS for additional $24 \mathrm{~h}$. iNOS protein level was determined by Western blot using a polyclonal antibody anti-iNOS (Ab160862, Cayman chemical). As control, a monoclonal anti- Tubulin (AbT6074, Sigma) has been used.

\section{Conclusions}

Overall, our results showed that pigmented rice varieties possessed high antioxidant capacity and high flavonoid and polyphenol content. Anthocyanins were mainly present in black rice, whereas alkylresorcinols were found only in red rice. Rice bran proved to be particularly rich in carotenoids. In terms of health benefits, we demonstrated that, upon inflammatory stimulus, extracts obtained from integral, black, red rice and bran specifically reduced the expression of iNOS, responsible for the NO production, without affecting cytokines secretion. These results indicate the capacity of rice extracts to modulate the inflammatory process, without abolishing the acute inflammatory response in case of pathogen infections.

\section{Acknowledgements}

This work was supported by the Fondazione Umberto Veronesi [Grant Fellowships to FT and VC]. The authors wish to thank Chiara Tonelli for helpful discussions, Euricom Spa for providing rice bran and Romano Gironi for assistance with the other rice materials.

\section{REFERENCES}

[1] E.M. Abdel-Aal, J.C. Young, I. Rabalski. Anthocyanin composition in black, blue, pink, purple, and red cereal grains. J Agric Food Chem, 54(13):4696-4704, 2006.

[2] A. Gunaratne, K. Wu, D. Li, A. Bentota, H. Corke, Y.-Z. Cai. Antioxidant activity and nutritional quality of traditional red-grained rice varieties containing proanthocyanidins. Food chemistry, 138(2):1153-1161, 2013.

[3] G.-F. Deng, X.-R. Xu, Y. Zhang, D. Li, R.-Y. Gan, H.-B. Li. Phenolic compounds and bioactivities of pigmented rice. Critical reviews in food science and nutrition, 53(3):296-306, 2013.

[4] F. Finocchiaro, B. Ferrari, A. Gianinetti, C. Dall'Asta, G. Galaverna, F. Scazzina, N. Pellegrini. Characterization of antioxidant compounds of red and white rice and changes in total antioxidant capacity during processing. Molecular nutrition \& food research, 51(8):1006-1019, 2007.

[5] M. Zaupa, L. Calani, D. Del Rio, F. Brighenti, N. Pellegrini. Characterization of total antioxidant capacity and (poly) phenolic compounds of differently pigmented rice varieties and their changes during domestic cooking. Food chemistry, 187:338-347, 2015.

[6] H. Chen, T. Siebenmorgen, K. Griffin. Quality characteristics of long-grain rice milled in two commercial systems. Cereal Chemistry, 75(4):560-565, 1998.

[7] G. Kennedy, B. Burlingame, N. Nguyen. Nutrient impact assessment of rice in major rice-consuming countries. International Rice Commission Newsletter, 2002.

[8] F.F. Paiva, N.L. Vanier, J.D.J. Berrios, V.Z. Pinto, D. Wood, T. Williams, J. Pan, M.C. Elias. Polishing and parboiling effect on the nutritional and technological properties of pigmented rice. Food chemistry, 191:105-112, 2016. 
[9] E.T. Champagne. Rice: chemistry and technology: American Association of Cereal Chemists; 2004.

[10] H.I. Jun, G.S. Song, E.I. Yang, Y. Youn, Y.S. Kim Antioxidant activities and phenolic compounds of pigmented rice bran extracts. Journal of food science, 77(7):C759-C764, 2012.

[11] A.M. Graves, N. Hettiarachchy, S. Rayaprolu, R. Li, R. Horax, H.S. Seo. Bioactivity of a Rice Bran-Derived Peptide and Its Sensory Evaluation and Storage Stability in Orange Juice. Journal of food science, 2016.

[12] M. Demont, E. Zossou, P. Rutsaert, M. Ndour, P. Van Mele, W. Verbeke. Consumer valuation of improved rice parboiling technologies in Benin. Food Quality and Preference, 23(1):63-70, 2012.

[13] Y.-T. Chiu, M.L. Stewart. Effect of variety and cooking method on resistant starch content of white rice and subsequent postprandial glucose response and appetite in humans. Asia Pacific journal of clinical nutrition, 22(3):372-379, 2013.

[14] C. Martin, Y. Zhang, C. Tonelli, K. Petroni. Plants, diet, and health. Annual review of plant biology, 64:19-46, 2013.

[15] R. Medzhitov. Origin and physiological roles of inflammation. Nature, 454(7203):428-435, 2008.

[16] ISO. http://www.iso.org/iso/home/store.htm, Accessed 06/01/2017.

[17] C. AACC. Approved methods of the American association of cereal chemists. Methods, 54:21, 2000.

[18] AOAC. Official methods of analysis of AOAC International: AOAC International; 2005.

[19] R. Re, N. Pellegrini, A. Proteggente, A. Pannala, M. Yang, C. Rice-Evans. Antioxidant activity applying an improved ABTS radical cation decolorization assay. Free radical biology and medicine, 26(9):1231-1237, 1999.

[20] A. Heredia, I. Peinado, E. Rosa, A. Andrés. Effect of osmotic pre-treatment and microwave heating on lycopene degradation and isomerization in cherry tomato. Food chemistry, 123(1):92-98, 2010.

[21] C. Simonelli, A. Abbiati, M. Cormegna. Physicochemical Characterization of some Italian rice varieties. The Journal of Food Science and Nutrition, 45(1):9-23, 2016.
[22] R. Angelini, A. Ferrero, I. Ponti. Il riso. Bayer Crop Science Edscript, Bologna, Italy:1-680, 2008.

[23] P. Suwannaporn, S. Pitiphunpong, S. Champangern. Classification of rice amylose content by discriminant analysis of physicochemical properties. Starch - Stärke, 59(3 4):171-177, 2007.

[24] L.J. Ells, C.J. Seal, B. Kettlitz, W. Bal, J.C. Mathers. Postprandial glycaemic, lipaemic and haemostatic responses to ingestion of rapidly and slowly digested starches in healthy young women. The British journal of nutrition, 94(6):948-955, 2005.

[25] G. Pereira-Caro, G. Cros, T. Yokota, A. Crozier. Phytochemical profiles of black, red, brown, and white rice from the Camargue region of France. Journal of agricultural and food chemistry, 61(33):7976-7986, 2013.

[26] A.B. Ross, M.J. Shepherd, M. Schüpphaus, V. Sinclair, B. Alfaro, A. Kamal-Eldin, P. Åman. Alkylresorcinols in cereals and cereal products. Journal of Agricultural and Food Chemistry, 51(14):4111-4118, 2003.

[27] A.B. Ross, A. Kamal-Eldin, P. Åman. Dietary Alkylresorcinols: Absorption, Bioactivities, and Possible Use as Biomarkers of Whole-grain Wheat-and Rye-rich Foods. Nutrition Reviews, 62(3):81-95, 2004.

[28] J.K. Kim, S.Y. Lee, S.M. Chu, S.H. Lim, S.-C. Suh, Y.-T. Lee, H.S. Cho, S.-H. Ha. Variation and correlation analysis of flavonoids and carotenoids in Korean pigmented rice (Oryza sativa L.) cultivars. Journal of agricultural and food chemistry, 58(24):12804-12809, 2010.

[29] S. Kong, J. Lee. Antioxidants in milling fractions of black rice cultivars. Food chemistry, 120(1):278-281, 2010.

[30] C. Hu, J. Zawistowski, W. Ling, D.D. Kitts. Black rice (Oryza sativa L. indica) pigmented fraction suppresses both reactive oxygen species and nitric oxide in chemical and biological model systems. J Agric Food Chem, 51(18):5271-5277, 2003.

[31] T.L. Wadsworth, D.R. Koop. Effects of Ginkgo biloba extract (EGb 761) and quercetin on lipopolysaccharide-induced release of nitric oxide. Chemico-biological interactions, 137(1):43-58, 2001.

[32] M. Xia, W.H. Ling, J. Ma, D.D. Kitts, J. Zawistowski. Supplementation of diets with the black rice pigment fraction attenuates atherosclerotic plaque formation in apolipoprotein e deficient mice. J Nutr, 133(3):744-751, 2003. 